UCM/CSIC-95-12

December 1995

\title{
The Correlated Block Renormalization Group
}

\author{
Miguel A. Martín-Delgado $\dagger$, Javier Rodriguez-Laguna $\ddagger$ and Germán Sierra* \\ †Departamento de Física Teórica I \\ Universidad Complutense. 28040-Madrid, Spain \\ $\ddagger$ Instituto de Matemáticas y Física Fundamental. C.S.I.C. \\ Serrano 123, 28006-Madrid, Spain \\ * Theoretische Physik - ETH-Honggerberg \\ CH-8093, Switzerland
}

\begin{abstract}
We formulate the standard real-space renormalization group method in a way which takes into account the correlation between blocks. This is achieved in a dynamical way by means of operators which reflect the influence on a given block of its neighbours. We illustrate our method in the example of the tight-binding model in 1D and 2D for various types of boundary conditions.
\end{abstract}

PACS numbers: 75.10 Jm, 05.50.+q, 64.60.Ak

${ }^{*}$ On leave of absence from Instituto de Matemáticas y Física Fundamental, C.S.I.C., Serrano 123, 28006-Madrid, Spain. 


\section{Introduction}

The initial success of the Real-Space Renormalization Group method applied by Wilson [1] to the study of the Kondo problem rised the hope that this technique could be generalizable to more complicated many-body problems in Field Theory and Condensed Matter. Various groups of physicists working on those areas were able to combine Wilson's ideas together with Kadanoff's concept of block thereby arriving to a method called Block Renormalization Group (BRG) [2], [3].

From a quantitative and sometimes qualitative point of view, the BRG procedure proved to be not fully reliable particularly when compared with numerical approaches, such as the Quantum Montecarlo method, which were developed at the same time. This was one of the reasons why the BRG methods remained undeveloped during the 80's until 1992 were a new numerical method called the Density Matrix Renormalization Group (DMRG) was proposed to overcome the difficulties of the old techniques [畔, [5].

In order to understand the failure of the BRG method Wilson proposed in 1986 [6] to study the problem of a single particle moving freely in a 1D box. A straightforward application of the BRG techniques gives results which are several orders of magnitude off the exact values. According to White and Noack [5] this poor performance is due to the fact that the truncation of states within each block in the BRG method keeps states which do not have the appropriate boundary conditions (BC). As a matter of fact, an isolated block has BC's which are different from those it has when it is immersed into the rest of the lattice. In [5] two solutions to this problem were proposed which in turn give rise to two different methods called Combination of Boundary Conditions (CBC) and the aforementioned DMRG [A]. In the CBC method one applies different BC's to each block and mix them up resulting in an accurate performance. However, this method is difficult to generalize for interacting systems. On the contrary, the DMRG method has been applied with great success to interacting systems [7], [8]. In the DMRG method each block somehow chooses the exact BC it needs. This is achieved by putting the block in connection with the rest of the lattice. In practical terms, one considers a superblock where the block is embedded, then one finds the exact ground state of the superblock (called the target state) and constructs a reduced density matrix $\rho_{B}$ for the block. This density matrix $\rho_{B}$ gives the best posible representation of the target state on the block. Finally, one diagonalizes $\rho_{B}$ and keeps the eigenstates with largest eigenvalues in the truncation procedure. In this fashion, the role of the Block Hamiltonian $H_{B}$ in the BRG method is played in the DMRG method by the density matrix, which has the virtue of containing the effect of the neighbourhood on a given block.

The DMRG method has been generalized to 2D classical systems in references [9], [10]. In this framework the density matrix turns out to be intimately related to the Baxter's corner transfer matrix 11], which suggests a pathway for a deeper understanding of the DMRG techniques. In reference [12], [13] we have introduced the Renormalization Quantum Group Method (qRG for short) based on quantum groups techniques and we mentioned that this qRG method has some analogies with the DMRG.

All these works show that the DMRG is not only a powerful computational method but also a source of inspiration for further works concerning the RG. For these reasons, it may be worthwhile to explore different options or alternatives to the DMRG which may be useful in situations where the DMRG encounters difficulties, as in the case of $2 \mathrm{D}$ quantum systems. The main message of the DMRG is that blocks are correlated. The implementation of this idea by means of the density matrix formalism may be not the unique way to proceed. On the other hand, the "onion-scheme" a la Wilson adopted by the DMRG, while being one of the reasons of its spectacular accuracy, imposes certain limitations.

At this stage it is not clear how fundamental are the density-matrix formalism or the onion-scheme for a RG method which takes into account the correlation between blocks. One can indeed combine the Kadanoff block method with the use of a density matrix in the process of truncation, as in reference [14. More work remains to be done to see wheather there is a real improvement of the standard 
BRG method by combining it with the DMRG as in [14]. In this paper we want to explore another possibility which is to give up both the density matrix and the onion-scheme. With this point of view in mind, it would seem that we should come pretty close to the standard BRG method, were it not for the enormous freedom hidden in a Real-Space RG method. This freedom comes from the separation of the Hamiltonian into an intrablock $H_{B}$ and an interblock $H_{B B}$ Hamiltonian. This is a source of ambiguities which can be sometimes mitigated with the aide of symmetry arguments, but not fully eliminated though. This ambiguity shows up specially for terms in the Hamiltonian acting at the boundaries of the block. There are no general criteria as to how to include this type of terms either into the intrablock or into the interblock Hamiltonians, or into both! For example, in the 1D Ising model in a transverse field (ITF model), a choice which preserves the selfduality of the model attributes some self-couplings to the $H_{B}$ and others to the interblock $H_{B B}$, and it yields to an exact value of the critical point and the critical exponent $\nu$ [16], [12]. The ambiguity in the splitting of $H$ into the sum $H_{B}+H_{B B}$ thus affect deeply the truncation procedure itself, which is based on the diagonalization of $H_{B}$. Rather than blaming the BRG for its lack of uniqueness, we should use its freedom to allow the blocks to become correlated in the RG procedure. In our present approach this correlation will be taken into account in a "dynamical" way rather than in a "statistical" way as in the DMRG. This will be achieved by the introduction of interblock operators which reflect the "influence" between neighbour blocks and which are defined at the boundary of the block in the first step of our CBRG method.

We have chosen to illustrate our approach the 1D and 2D tight-binding models mainly for simplicity reasons, but we believe that our method could be applied to more complicated problems. In fact, the first step in this direction was already undertaken in reference 15], where only 2 states at each stage of the RG-blocking were retained. This in turn allowed us to obtain the $1 / N^{2}$ scaling law for the size dependence of the first-excited-state energy.

In this paper we shall give the general mathematical structure underlying the results of reference [15]. This will allow us to retain more than two states in the RG-truncation and also to consider the two-dimensional tight-binding model. In this fashion, we shall recover the $n^{2} / N^{2}$ scaling law for the

$n$-th excited state of the $1 \mathrm{D}$ model and the scaling law $\frac{n_{1}^{2}+n_{2}^{2}}{N^{2}}$ in the $2 \mathrm{D}$ case. These results will then show that the CBRG method describes correctly the low energy behaviour of the 1D and 2D Laplacian.

This paper is organized as follows. In Sect.2 we present the Correlated Block RG-method and apply it to the 1D Tight-Binding model with different boundary conditions at the ends of the chain: Free-Free, Free-Fixed and Fixed-Fixed BC's. We correctly reproduce in each and every case the corresponding $n^{2} / N^{2}$-scaling laws $(N \longrightarrow \infty)$ for the spectrum of the excited states. This is a novel result for it is achieved within the framework of the CBRG method in a clear and transparent fashion without having to resort to ad hoc mixing of different BC's states in the truncation operator involved in the CBC method [5]. In Sect.3 we extend the CBRG method to deal with two-dimensional Hamiltonians and apply the procedure to successfully solve the 2D tight-binding model with Free BC's. Sect.4 is devoted to conclusions and prospectives.

\section{The CBRG Method: One Dimension}

The problem we want to study is the one-dimensional Tight-Binding model in an open chain with different boundary conditions at its ends. The Hamiltonian for this system takes the following matricial form, 


$$
H_{b, b^{\prime}}=\left(\begin{array}{cccccc}
b & -1 & & & & \\
-1 & 2 & -1 & & & \\
& -1 & 2 & & & \\
& & & \ddots & & \\
& & & & 2 & -1 \\
& & & & -1 & b^{\prime}
\end{array}\right)
$$

where $b$ and $b^{\prime}$ take on the values 1 (or 2) corresponding to Free (or Fixed) BC's respectively. This Hamiltonian is the discrete version of the Laplacian $H=-\partial_{x}^{2}$, while the Free or Fixed BC's correspond in the continuum to the vanishing of the wave function (Fixed BC's) or its spatial derivative (Free BC's) at the ends of the chain, i.e.,

$$
\begin{array}{llc}
b=2 & \Rightarrow \quad \Psi(0)=0 & \text { Fixed BC } \\
b=1 \quad \Rightarrow \quad \frac{\partial \Psi}{\partial x}(0)=0 & \text { Free BC }
\end{array}
$$

and similarly for $b^{\prime}$ which contains the $\mathrm{BC}$ at the other end of the chain.

Hence, altogether there are 4 Hamiltonians of the type in (2.1), whose eigenstates and eigenvalues are the subject of our RG-techniques.

The first step in the RG method is to divide the lattice into blocks containing $n_{s}$ sites each and labeled with and index $p\left(=1, \ldots, N / n_{s}\right)$. Let us suppose for a moment that we isolate the $p$ th-block from the rest of the lattice so that its dynamics, as an independent entity, is governed by a Hamiltonian denoted by $A_{p}$, which we may call uncorrelated block Hamiltonian. The restoration of the block back into the lattice involves two effects. The first one is that the BC's of the $p$-th block may change under the influence of the $p+1$ and $p-1$ blocks. We describe this change of BC's by the action of Boundary Operators denoted by $B_{p, p \pm 1}$ on the $p$ th-block. The second effect is the interaction between the $p$ thblock and its neighbours $p+1$ and $p-1$, given by interaction Hamiltonians $C_{p, p \pm 1}$ which act on both $p$ and $p+1$ blocks simultaneously. If the problem under consideration is translationally invariant, all the Hamiltonians defined above are independent of the block label $p$, in which case we denote them by,

$$
\begin{array}{cc}
A_{p}=A & \\
B_{p, p+1}=B_{R} & B_{p, p-1}=B_{L} \\
C_{p, p+1}=C & C_{p, p-1}=C^{\dagger}
\end{array}
$$

The $H_{\text {Free,Free }}$ Hamiltonian (2.1) gives an example of this as we shall show below. Hence, for the time being, we shall consider the situation described by (2.3) and leave the more general case after explaining the general ideas.

In the standard BRG method the block Hamiltonian $H_{B}$ and the interblock Hamiltonian $H_{B B}$ are given, according to our previous definitions, by the following formulas

$$
\begin{aligned}
& H_{B}=A+B_{L}+B_{R} \\
& H_{B B}=\left(\begin{array}{cc}
0 & C \\
C^{\dagger} & 0
\end{array}\right)
\end{aligned}
$$

The whole Hamiltonian is by all means the sum of $H_{B}$ and $H_{B B}$ for all the blocks of the chain. For a review on the Block RG method see [18] and chapter 11 of reference [19].

Next step in the RG method is to diagonalize $H_{B}$ and keep its, say $m\left(m<n_{s}\right)$, lowest eigenstates. The truncation is given by a $n_{s} \times m$ matrix $T$ whose columns are precisely the components of the $m$ lowest eigenstates of $H_{B}$. The renormalized Hamiltonian in the new basis is given by,

$$
H^{\prime}=T^{\dagger}\left(H_{B}+H_{B B}\right) T
$$


At first sight from Eq. (2.4) it would seem that we have taken into account the effect of the BC's on a given block. However, as the examples show, this is quite a bit illusory. On the other hand, the distinction among $A, B_{L}$ and $B_{R}$ is rather inmaterial as far as $H_{B}$ is concerned, and in fact no distinction of this sort is made in the standard BRG formalism. Finally, let us observe that $H_{B}$ and $H_{B B}$ play rather different roles in the truncation procedure. This asymmetry has been observed as a source of problems by several authors in the past [16], [17].

We shall mention that this asymmetry has recently been related to quantum groups in a fashion which has led to a new RG method called the Renormalization Quantum Group method [12], [13].

Therefore, from various points of view, one is urged to make more explicit the role played by the BC-operators $B_{L}$ and $B_{R}$ in our CBRG procedure. For this purpose, we have found convenient to use the concept of superblock already introduced in reference [5]. We shall define a superblock as the set of two consecutive blocks, $p$ and $p+1$ and denoted by $(p, p+1)$. The great advantage of the superblock is that it allows us to materialize the distinction among $A, B_{L}$ and $B_{R}$. In fact, just as the isolation of a single block leads us to the definition of the Hamiltonian $A$, the isolation of two blocks contained in a superblock allows us to define $B_{L}, B_{R}$ and also $C$ through the superblock Hamiltonian $H_{s B}$ as follows,

$$
H_{s B}=\left(\begin{array}{cc}
A+B_{R} & C \\
C^{\dagger} & A+B_{L}
\end{array}\right)
$$

Similarly, the Hamiltonian describing the interaction between superblocks is given by (see Fig.1)

$$
H_{s B, s B}=\left(\begin{array}{cccc}
0 & & & \\
& B_{R} & C & \\
& C^{\dagger} & B_{L} & \\
& & & 0
\end{array}\right)
$$

Now instead of diagonalizing $H_{B}$ in Eq. (2.4), in the CBRG method we shall diagonalize $H_{s B}$ in Eq. (2.7), and afterwards keep the $m=n_{s}$ lowest eigenstates in the tight-binding model. As in the standard $\mathrm{BRG}$ method, the change to the truncated basis defines the renormalized operators as follows:

$$
\begin{gathered}
H_{s B} \longrightarrow T^{\dagger} H_{s B} T=A^{\prime} \\
H_{s B, s B} \longrightarrow T^{\dagger} H_{s B, s B} T=\left(\begin{array}{cc}
B_{R}^{\prime} & C^{\prime} \\
C^{\prime \dagger} & B_{L}^{\prime}
\end{array}\right)
\end{gathered}
$$

where the matrices $A^{\prime}, B_{R}^{\prime}, B_{L}^{\prime}$ and $C^{\prime}$ are the renormalized version of the operators $A, B_{R}, B_{L}$ and $C$, and they exhibit the same geometrical interpretation for the renormalized block as their unprimed partners for the original blocks.

If we set $B_{R}=B_{L}=0$ in Eqs. (2.7) and (2.8), then after the first RG-step we get $B_{R}^{\prime}=B_{L}^{\prime}=0$ and thus the previous RG-scheme coincides with the standard BRG. We may say that uncorrelated blocks are in a sense a fixed point of our method. However, this fixed point may be unstable, and to explore this possibility one has to look for non-vanishing $B$-operators and their RG-evolution.

Let us address now some examples. We shall first study the Hamiltonian (2.1) with Free BC's at the ends $\left(b=b^{\prime}=1\right)$. Choosing $n_{s}=3$ for example, we see that the choice for the operators $A, B_{R}, B_{L}$ and $C$ in the first step of the CBRG procedure is given by,

$$
A=\left(\begin{array}{ccc}
1 & -1 & 0 \\
-1 & 2 & -1 \\
0 & -1 & 1
\end{array}\right), B_{R}=\left(\begin{array}{lll}
0 & & \\
& 0 & \\
& & 1
\end{array}\right), B_{L}=\left(\begin{array}{ccc}
1 & & \\
& 0 & \\
& & 0
\end{array}\right), C=\left(\begin{array}{ccc}
0 & 0 & 0 \\
0 & 0 & 0 \\
-1 & 0 & 0
\end{array}\right)
$$


This choice is equivalent to the assumption that an isolated block satisfies Free BC's at its ends. The role of $B_{R}$ and $B_{L}$ is to join these blocks into a single chain. This is the geometrical explanation of Eqs. (2.11). In more general cases one must have to explore which is the best choice. The generalization of Eqs. 2.11) to blocks with more than 3 sites is obvious. In Table 1 we collect our CBRG-results for the first 5 excited states for a chain of $N=12 \times 2^{6}=768$ sites. Comparison with exact results gives a good agreement.

An important feature of our CBRG method is that the $n^{2} / N^{2}$ - scaling law $(N \longrightarrow \infty)$ for the energy of the $n$-excited states of a chain made up of $N$ sites, is reproduced correctly (see Fig.2). In Table 2 we show the variation of the first-excited-state energy with the size $N$ of the chain. From those values we can extract the corresponding $1 / N^{2}$-law which turns out to be,

$$
E_{1}^{(C B R G)}(N)=c_{C B R G}^{(1)} \frac{1}{N^{2}}, \quad c_{C B R G}^{(1)}=9.8080,(N \longrightarrow \infty) \text { Free-Free BC's }
$$

while the exact value for the proportionality constant $c$ is $c_{\text {exact }}=\pi^{2}=9.86$. This amounts to a 0.6 $\%$ error.

Likewise, we have enough data so as to obtain the corresponding $n^{2} / N^{2}$-law for the whole set of 5 excited states. Thus, the scaling law we obtain is,

$$
E_{n}^{(C B R G)}(N)=c_{C B R G} \frac{n^{2}}{N^{2}}, \quad c_{C B R G}=8.4733,(N \longrightarrow \infty) \text { Free-Free BC's }
$$

which now amounts to a $7.34 \%$ error. This is a natural fact from the worse knowledge of the highest excited states of the spectrum in a RG-scheme.

We can make even more explicit the successful achievement of the $1 / N^{2}$-scaling law by leaving as a free adjustable parameter the exponent of $1 / N$ in addition to the proportionality constant. Let us denote by $\theta$ this critical exponent. Using data from 20 to 50 steps of our CBRG-method for several truncation of states according to our scheme $2 n_{s} \rightarrow n_{s}$ (namely, $n_{s}=10,13,20$ ) we arrive at the following results,

$$
\begin{aligned}
E_{1}^{(C B R G)}(N)=c_{C B R G} \frac{1}{N^{\theta}}, & (N \longrightarrow \infty) \text { Free-Free BC's } \\
& \begin{aligned}
\text { For } 20 \longrightarrow 10 & \theta=1.9708 \\
\text { For } 26 \longrightarrow 13 & \theta=1.9734 \\
\text { For } 40 \longrightarrow 20 & \theta=1.9854
\end{aligned}
\end{aligned}
$$

These results clearly support the fact that we have correctly reproduced the exact value of $\theta=2$ for the finite-size critical exponent.

Last, but not least, as was proved in [15] our CBRG method gives the exact energy of the ground state for every step of the RG-procedure for Free-Free BC's.

In reference [15] it was shown that one can reproduce easily the wave function of the excited states. This procedure was called reconstruction since it works "downwards" in the CBRG method. The basic equation to be used is the reconstruction equation [15],

$$
\Psi^{(r+1)}=L_{r} \Psi_{L}^{(r)}+R_{r} \Psi_{R}^{(r)}
$$

where $\Psi^{(r)}$ denotes the collection of $m$ lowest eigenstates in the $r$-step of the CBRG-procedure, and $L_{r}$, $R_{r}$ are the block matrices in terms of which the truncation matrix $T^{\dagger}$ can be written as $T^{\dagger}=\left(L_{r}, R_{r}\right)$. Our results for a chain of $N=12 \times 2^{6}=768$ sites and $n_{s}=6$ states kept are given in Fig. 3 where we have plotted the first 5 excited states and compare them with the exact wave functions. There are some remarkable facts regarding these figures. Firstly, the number of nodes is correctly preserved by our CBRG wave functions. Secondly, the Free-Free type of boundary conditions are also correctly 
reproduced at the ends of the chain. And lastly, it is worthwhile to point out that the CBRG wave functions "degrade gracefully" as the energy of the excited state raises in accordance with the fact that the lower the energy is, the more reliable are the results.

This ends the results for the Free-Free BC's. In order to address other types of BC's we must come back to the case where the matrices $A, B_{R}, B_{L}$ and $C$ depend on each particular block. Thus, for example, for the Fixed-Free BC's we shall choose as the uncorrelated $A$-matrix for the block located to the left end of the chain the following form $\left(n_{s}=3\right)$,

$$
A_{1}=\left(\begin{array}{ccc}
2 & -1 & 0 \\
-1 & 2 & -1 \\
0 & -1 & 1
\end{array}\right) \quad \text { Fixed-Free BC's }
$$

while the remaining matrices $A_{p},(p=2, \ldots, N / 3)$, will be given by Eqs.2.3), 2.11).

For Free-Fixed BC's, it is the last $A$-matrix which we have to take different from the others, namely,

$$
A_{N / 3}=\left(\begin{array}{ccc}
1 & -1 & 0 \\
-1 & 2 & -1 \\
0 & -1 & 2
\end{array}\right) \text { Free-Fixed BC's }
$$

As for the Fixed-Fixed BC's case, we must change the $A$-matrix at both ends of the chain according to the following prescription,

$$
A_{1}=\left(\begin{array}{ccc}
2 & -1 & 0 \\
-1 & 2 & -1 \\
0 & -1 & 1
\end{array}\right), \quad A_{N / 3}=\left(\begin{array}{ccc}
1 & -1 & 0 \\
-1 & 2 & -1 \\
0 & -1 & 2
\end{array}\right) \text { Fixed-Fixed BC's }
$$

Then we follow the same steps as for the Free-Free BC's, taking care that the $A, B_{R}, B_{L}$ and $C$ matrices in each CBRG-step may depend on the position of the blocks. This implies in particular that the embedding $T$-matrices may also vary from block to block.

In Tables 3 and 4 we summarize our results for the Free-Fixed and Fixed-Fixed BC's (Fixed-Free BC's are equivalent to Free-Fixed BC's by parity transformation). In these tables we present our CBRG results for the first 6 lowest lying states for the 1D tight-binding model in a chain of $N=12 \times 2^{5}=384$ sites with mixed boundary conditions, and they are compared against the exact and standard BRG values. Several remarks are in order. First, we observe that the CBRG method produces a good agreement with the exact results and certainly much more accurate by several orders of magnitude than the old BRG method. Second, the CBRG method is able to reproduce the corresponding $n^{2} / N^{2}$ scaling laws for the spectrum of excited states in each case of mixed BC's. Namely,

- For Free-Fixed BC's and considering just the ground state, we have

$$
E_{0}^{(C B R G)}(N)=c_{C B R G}^{(0)} \frac{1}{4 N^{2}}, \quad c_{C B R G}^{(0)}=9.072,(N \longrightarrow \infty) \text { Free-Fixed BC's }
$$

which amounts to a $8 \%$ error with respect to the exact value of $c_{\text {exact }}=\pi^{2}$.

As for the corresponding law for the whole spectrum, we find

$$
E_{n}^{(C B R G)}(N)=c_{C B R G} \frac{(n+1)^{2}}{4 N^{2}}, \quad c_{C B R G}=7.6729,(N \longrightarrow \infty) \text { Free-Fixed BC's }
$$

which represents a $11.5 \%$ error with respect to the exact value of $\pi^{2}$. 
- For Free-Fixed BC's and considering just the ground state, we have

$$
E_{0}^{(C B R G)}(N)=c_{C B R G}^{(0)} \frac{1}{N^{2}}, \quad c_{C B R G}^{(0)}=8.35,(N \longrightarrow \infty) \text { Fixed-Fixed BC's }
$$

which amounts to a $8 \%$ error with respect to the exact value of $c_{\text {exact }}=\pi^{2}$.

As for the corresponding law for the whole spectrum, we find

$$
E_{n}^{(C B R G)}(N)=c_{C B R G} \frac{(n+1)^{2}}{N^{2}}, \quad c_{C B R G}=6.9696,(N \longrightarrow \infty) \text { Fixed-Fixed BC's }
$$

which represents a $16 \%$ error with respect to the exact value of $\pi^{2}$.

We obtain bigger errors in the determination of these scaling laws as compared with the Free-Free case mainly because we have used less data in our fitting. Nevertherless, we find a good agreement with the exact results. Yet, there is another reason as to why the accuracy in the case of mixed BC's is worse, namely, the ground state wave function $\Psi_{0}$ is not homogeneous in space as it is in the Free-Free case [15]. This makes the RG-procedure more involved and a source of extra uncertainties.

Let us mention in passing that we are also able to make a wave function reconstruction in the mixed BC's cases as has been done for the Free-Free BC case.

The outcome of all the results presented so far is that we have succeded in devising a Real-Space RG method capable of reproducing the correct eigenvalues and eigenstates for the tight-binding model as originally envisaged by Wilson, within a certain accuracy which can in principle be improved.

Althoug the model we have employed to test our CBRG-method is a tight-binding model, there are some remarkable facts regarding the fixed-point structure of our CBRG-solution that we would like to stress. Namely, we have found that after enough number of CBRG-iterations, the matrices $A, B_{L}, B_{R}$ and $C$ in the Free-Free case scale nicely with the size $N$ of the chain according to the dynamical critical exponent $z$. To be more precise, let us introduce the fixed point values of those matrices denoted by $A^{*}, B_{L}^{*}, B_{R}^{*}$ and $C^{*}$ which we define as,

$$
A^{*}=N^{-z} a^{*}, \quad B_{L}^{*}=N^{-z} b_{L}^{*}, \quad B_{R}^{*}=N^{-z} b_{R}^{*}, \quad C^{*}=N^{-z} c^{*}, \quad \text { Fixed-Point values }
$$

in terms of the scaled matrices $a^{*}, b_{L}^{*}, b_{R}^{*}$ and $c^{*}$. For a block of 3 sites $\left(n_{s}=3\right)$ we find the following Fixed-Point structure parametrized by two constants $s$ and $t$ (for bigger $n_{s}$ we need extra parameters),

$$
a^{*}=0, b_{R}^{*}=\left(\begin{array}{ccc}
1 & s & s \\
s & t & t \\
s & t & t
\end{array}\right), b_{L}^{*}=\left(\begin{array}{ccc}
1 & -s & s \\
-s & t & -t \\
s & -t & t
\end{array}\right), c^{*}=\left(\begin{array}{ccc}
-1 & s & -s \\
-s & t & -t \\
-s & t & -t
\end{array}\right)
$$

with $s=1.3993$ and $t=1.9581$. The critical exponent $z$ we obtain is,

$$
z=0.9999
$$

which is indeed very close to the exact value $z=1$ (actually, it differs in the ninth decimal digit). The interpretation of this Fixed-Point in the context of the CBRG method is as follows. We pointed out before that when the boundary operators $b_{L, R}$ vanish we recover the standard BRG method in which the blocks are not correlated. Here we find that it is the uncorrelated Hamiltonian which vanish, while the boundary $b_{L, R}$ and interaction $c$ operators do not vanish within the scaling law. This fact may perhaps be interpreted by saying that in the example under study the correlation between blocks is more important than their selfenergy. In references [9], [10], [11] it was shown that the DMRG method leads, in the thermodynamic limit, to a "product form" ansatz for the ground state wave function. In our case we see from Eqs.(2.24), (2.25) that we also reach thermodynamical limit, which leads us to ask 
about the nature of the ansatz for the ground state and excited states implied by the CBRG method. The answer to this question will be addressed in a future publication but it suffices to say that both the DMRG and the CBRG methods seem to yield different ansatzs of the ground state wave function. In a few words, the DMRG is associated with a "vertex picture" while the CBRG is associated with a "string picture".

\section{The Two-Dimensional CBRG-Algorithm}

The RG-method that we have devised in the one-dimensional problem can be generalized in a natural way to higher dimensions. We shall consider for simplicity the $2 \mathrm{D}$ case. First of all, we divide the square lattice into blocks of $n_{s}$ sites each. Each block will in turn be a square lattice with a minimum of 4 sites $(=2 \times 2$ block $)$. As in $1 \mathrm{D}$, we shall define the following Hamiltonians to carry out the CBRG-program,

- $A_{p}=$ self-energy of the $p$-th block isolated from the lattice.

- $B_{p, q}=$ self-energy of the $p$-th block induced by the presence of the $q$-th block.

- $C_{p, q}=$ interaction between the $p$-th block and the $q$-th block.

The difference with respect to the 1D case is that each block has now 4 neighbours and therefore there are four different $B$ and $C$ matrices.

Let us consider again the Hamiltonian of a free particle moving in a 2D-box with Free BC's at the boundaries of the box. The 2D Hamiltonian is given again by the incidence matrix of the lattice. As in $1 \mathrm{D}$ we shall choose the matrix $A$ as the incidence matrix of the block. Thus, for example, for a $2 \times 2$ block labelled as in Fig.4 we have,

$$
A=\left(\begin{array}{cccc}
2 & -1 & 0 & -1 \\
-1 & 2 & -1 & 0 \\
0 & -1 & 2 & -1 \\
-1 & 0 & -1 & 2
\end{array}\right)
$$

The 4 Boundary Operators $B$ in the same basis as in Fig.5 a have a diagramatic representation as shown in Fig.4 which helps us to keep track of their location in the block $H_{B}$ and interblock $H_{B B}$ Hamiltonians. Dotted line in Fig.4 means influence or correlation between neighbouring blocks. Their explicit matricial form is as follows,

$$
\begin{aligned}
& B_{12}=B_{43}=B_{L}=\left(\begin{array}{cccc}
0 & & & \\
& 1 & & \\
& & 1 & \\
& & & 0
\end{array}\right), \quad B_{21}=B_{34}=B_{R}=\left(\begin{array}{cccc}
1 & & & \\
& 0 & & \\
& & 0 & \\
& & & 1
\end{array}\right) \\
& B_{14}=B_{23}=B_{D}=\left(\begin{array}{cccc}
0 & & & \\
& 0 & & \\
& & 1 & \\
& & & 1
\end{array}\right), \quad B_{41}=B_{32}=B_{U}=\left(\begin{array}{cccc}
1 & & & \\
& 1 & & \\
& & 0 & \\
& & 0
\end{array}\right)
\end{aligned}
$$

where the labels denote the position of the neighbouring blocks and we have used the translation invariance of the 2D tight-binding model so that we need only to distinguish between Right and Left, and Up and Down.

As for the Interaction $C$-Operators whose diagramatic representation is depicted in Fig.4 we have the following matricial representation, with the same considerations as for the $B$-operators, 


$$
\begin{aligned}
& C_{12}=C_{43}=C_{L R}=\left(\begin{array}{cccc}
0 & 0 & 0 & 0 \\
-1 & 0 & 0 & 0 \\
0 & 0 & 0 & -1 \\
0 & 0 & 0 & 0
\end{array}\right), C_{21}=C_{34}=C_{R L}=\left(\begin{array}{cccc}
0 & -1 & 0 & 0 \\
0 & 0 & 0 & 0 \\
0 & 0 & 0 & 0 \\
0 & 0 & -1 & 0
\end{array}\right) \\
& C_{14}=C_{23}=C_{D U}=\left(\begin{array}{cccc}
0 & 0 & 0 & 0 \\
0 & 0 & 0 & 0 \\
0 & -1 & 0 & 0 \\
-1 & 0 & 0 & 0
\end{array}\right), C_{41}=C_{32}=C_{U D}=\left(\begin{array}{cccc}
0 & 0 & 0 & -1 \\
0 & 0 & -1 & 0 \\
0 & 0 & 0 & 0 \\
0 & 0 & 0 & 0
\end{array}\right)
\end{aligned}
$$

Thus translation invariance reduces the number of independent CBRG-matrices by a half. These relations are particular of the problem at hand but we must left open the posibility of having all those matrices different from each other in order to handle more complicated problems.

Now that we have all the elements entering in our CBRG-method we proceed to construct the block $H_{s B}$ and interblock $H_{s B, s B}$ Hamiltonians out of them. To this end we have to consider a superblock made up of 4 blocks as shown in Fig.5 a) where the basis chosen is made explicitly. Thus, for $H_{s B}$ we have,

$$
H_{s B}=\left(\begin{array}{cccc}
A+B_{L}+B_{D} & C_{L R} & 0 & C_{D U} \\
C_{R L} & A+B_{R}+B_{D} & C_{D U} & 0 \\
0 & C_{U D} & A+B_{R}+B_{U} & C_{R L} \\
C_{U D} & 0 & C_{L R} & A+B_{L}+B_{U}
\end{array}\right)
$$

This is a $4 n_{s} \times 4 n_{s}$ matrix made up of $n_{s} \times n_{s}$ matrices.

As for the interblock Hamiltonian $H_{s B, s B}$ we have to distinguish between $(s B, s B)$-couplings of horizontal type denoted by $H_{s B, s B}^{(h o r)}$ and vertical type denoted by $H_{s B, s B}^{(v e r)}$, which read explicitly as,

$$
\begin{aligned}
H_{s B, s B}^{(h o r)} & =\left(\begin{array}{ccccccccc}
B_{R} & & & & 0 & C_{R L} & 0 & 0 \\
& 0 & & & 0 & 0 & 0 & 0 \\
& & 0 & & 0 & 0 & 0 & 0 \\
& & & B_{R} & 0 & 0 & C_{R L} & 0 \\
0 & 0 & 0 & 0 & 0 & & & \\
C_{L R} & 0 & 0 & 0 & & B_{L} & & \\
0 & 0 & 0 & C_{L R} & & & B_{L} & \\
0 & 0 & 0 & 0 & & & & 0
\end{array}\right) \\
H_{s B, s B}^{(v e r)}= & \left(\begin{array}{ccccccccc}
B_{D} & & & & & 0 & 0 & 0 & C_{U D} \\
& B_{D} & & & 0 & 0 & C_{U D} & 0 \\
0 & 0 & 0 & 0 & 0 & & & \\
0 & 0 & 0 & 0 & & 0 & & \\
0 & C_{D U} & 0 & 0 & & & B_{U} & \\
C_{D U} & 0 & 0 & 0 & & & & B_{U}
\end{array}\right)
\end{aligned}
$$

where we have made use again of translational invariance.

Once that we have made our choice for the decomposition of the total Hamiltonian of the 2D-tightbinding model into block and interblock Hamiltonians according to our CBRG-prescription, we can 
carry on with the truncation part of the RG-method. We shall keep $n_{s}$ states out of $4 n_{s}$ states per superblock so that our truncation scheme may be summarized as,

$$
4 n_{s} \text { (superblock) } \longrightarrow n_{s} \text { (new block) }
$$

Recall that at each step of the CBRG-method we need to identify the $A, B_{L}, B_{R}$ and $C$ operators which define the truncation procedure for the next step of the method. For this purpose, firstly the truncation of the superblock $H_{s B}$ gives rise to the $A^{\prime}$ uncorrelated self-energy operator for the next RG-step, namely,

$$
H_{s B}\left(4 n_{s} \times 4 n_{s} \text { matrix }\right) \longrightarrow A^{\prime}\left(n_{s} \times n_{s} \text { matrix }\right)
$$

To identify the rest of the operators we have to renormalize the interblock Hamiltonian which comes in two types, horizontal and vertical. As exemplified schematically in Fig.6 the renormalization of the $H_{s B, s B}^{(h o r)}$ Hamiltonian is given by,

$$
H_{s B, s B}^{(h o r)} \longrightarrow\left(\begin{array}{cc}
B_{R}^{\prime} & C_{R L}^{\prime} \\
C_{L R}^{\prime} & B_{L}^{\prime}
\end{array}\right)
$$

Likewise, for the $H_{s B, s B}^{(v e r)}$ Hamiltonian we have (see Fig.6),

$$
H_{s B, s B}^{(v e r)} \longrightarrow\left(\begin{array}{cc}
B_{D}^{\prime} & C_{U D}^{\prime} \\
C_{D U}^{\prime} & B_{U}^{\prime}
\end{array}\right)
$$

Now that we have identified all the operators defining the CBRG method at the new stage of the renormalization, we may reconstruct the new superblock Hamiltonian $H_{s B}^{\prime}$, which in turn has the same form as the original $H_{s B}$ in Eq.(3.6) substituting all the operators by their primed versions. This statement can be explicitly checked by considering the set of 4 superblocks as depicted in Fig.5. Firstly, the new $H_{s B}^{\prime}$ has a contribution coming from the truncation of each of the 4 superblocks in Fig.5, each of them contributing with an $A^{\prime}$-operator as in Eq.(3.9). Secondly, $H_{s B}^{\prime}$ picks up two more contributions coming from the horizontal and vertical interaction between superblocks, which we denote by $H_{\leftrightarrow}$ and $H_{\uparrow}$. Thus, in the CBRG-method $H_{s B}^{\prime}$ is renormalized as,

$$
\begin{aligned}
& \left.H_{s B}^{\prime}=\left(\begin{array}{cccc}
A^{\prime} & & & \\
& A^{\prime} & & \\
& & A^{\prime} & \\
& & & A^{\prime}
\end{array}\right) \longleftarrow \text { (single superblock contribution }\right) \\
& \left(H_{\leftrightarrow}\right) \rightarrow+\left(\begin{array}{cccc}
B_{L}^{\prime} & C_{L R}^{\prime} & & \\
C_{R L}^{\prime} & B_{R}^{\prime} & & \\
& & 0 & \\
& & & 0
\end{array}\right)+\left(\begin{array}{cccc}
0 & & & \\
& 0 & & \\
& & B_{R}^{\prime} & C_{R L}^{\prime} \\
& & C_{L R}^{\prime} & B_{L}^{\prime}
\end{array}\right) \\
& \left(H_{\uparrow}\right) \rightarrow+\left(\begin{array}{cccc}
B_{U}^{\prime} & & & C_{D U}^{\prime} \\
& 0 & & \\
& & 0 & \\
C_{U D}^{\prime} & & & B_{D}^{\prime}
\end{array}\right)+\left(\begin{array}{cccc}
0 & & & \\
& B_{U}^{\prime} & C_{D U}^{\prime} & \\
& C_{U D}^{\prime} & B_{D}^{\prime} & \\
& & & 0
\end{array}\right)
\end{aligned}
$$

and altogether we arrive at the previously stated result of Eq.(3.6).

Similarly we may proceed with the renormalized interblock Hamiltonians ${H^{\prime}}_{s B, s B}^{(\text {hor }}(\mathbf{3 . 1 0})$ and ${H^{\prime}}_{s B, s B}^{(v e r)}$ (3.11) and we end up with the same form for them as the original ones.

This ends the implementation of the CBRG-method for the 2D-tight-binding model. 
In Table 5 we collect our CBRG results for the first 4 lowest lying states for a chain of $N=$ $4 \times 4 \times 4^{6}=65536$ sites. Comparison with the exact results gives a good agreement. We have also data from truncations with blocks of $n_{s}=9$ and $n_{s}=16$ sites which enforce this statement. Moreover, notice that the first excited state is a doublet as in the exact solution.

Another important result of our CBRG-method is that the $\left(n_{1}^{2}+n_{2}^{2}\right) / N^{2}$ scaling law for the energy of the $\left(n_{1}, n_{2}\right)$-excited states of a square lattice of length $N$ is reproduced correctly. In fact, from data of the $n_{s}=16$ sites truncation for the first-excited-state energy we can extract the corresponding $1 / N^{2}$-scaling law which turns out to be,

$$
E_{1}^{(C B R G)}(N)=c_{C B R G}^{(1)} \frac{1}{N^{2}}, \quad c_{C B R G}^{(1)}=9.7365,(N \longrightarrow \infty) \mathrm{D}=2 \text { Free BC's }
$$

while the exact value of the proportionality constant $c$ is $c_{\text {exact }}=\pi^{2}=9.86$. This amounts to a $1.3 \%$ error.

Likewise, we may obtain the full $\left(n_{1}^{2}+n_{2}^{2}\right) / N^{2}$ scaling law for the whole set of 15 excited states and we find,

$$
E_{\left(n_{1}, n_{2}\right)}^{(C B R G)}(N)=c_{C B R G}^{(1)} \frac{\left(n_{1}^{2}+n_{2}^{2}\right)}{N^{2}}, \quad c_{C B R G}=7.9074,(N \longrightarrow \infty) \mathrm{D}=2 \text { Free BC's }
$$

which now amounts to a $10.5 \%$ error.

As in the 1D Free-Free case, we can determine critical scaling exponent $\theta$ (2.14). For a truncation scheme $16 \rightarrow 4$ we find,

$$
\theta=1.99999981 \quad \mathrm{D}=2
$$

which clearly supports the scaling laws introduced above. Notice again (see Table 5) that our CBRG method gives the exact (within machine precision) energy of the ground state. This is true for every step of the RG, as was proved in [15] for 1D.

We can also perform the wave function reconstruction of the excited states in the two-dimensional real space. This is achieved by a two-dimensional extension of the reconstruction equation (2.16). As an illustration of how the CBRG method performs with this matter, in Fig.7 we show a two-dimensional density plot of the wave function reconstruction for the siglet $(1,1)$-excited state in a square lattice of $N=65536$ sites with Free BC's. In that figure we show level lines separating regions of different height which are colored differently (darker grey levels meaning higher height). This density plot is made by projecting the $3 \mathrm{D}$ wave function representation onto the $x-y$ plane according to the height of the function. Thus, we may appreciate two maxima (black spots) and two minima (white spots) at the corners of the lattice, as it should be according to the exact solution.

Recall that the exact solution for the $(1,1)$ state has two lines of nodes, one vertical and one horizontal, which pass through the mid-points of each lattice side. These two node-lines are the analogue of the node-points of the 1D excited states in Fig.3. We have also obtained these node-lines correctly within our method. Thus, the qualitative real-space form of the excited-state wave functions are captured by the CBRG procedure.

\section{Conclusions and Prospectives}

We have presented in this paper a new Real-Space Renormalization Group method called Correlated Block RG (CBRG) which has been very much inspired by the Density Matrix RG method introduced by White [ [4], [5]. The basic notion developed by White in his novel treatment of Real-Spaces RG methods is that blocks are not isolated during the process of truncation of states and that they are certainly correlated. We have made a novel treatment of this concept without having to resort to a density matrix formalism nor to Wilson's onion-scheme for enlarging the lattice (chain). 
The way in which we take into account correlation between blocks is a dynamical one. This means that we start the first step of our CBRG method defining uncorrelated block Hamiltonians $A$, boundary operators $B$ and interaction operators $C$ which are used to construct the block Hamiltonian $H_{B}$ and thereby the truncation operator $T^{\dagger}$. This construction is reproduced at each step of the RG procedure. Thus, it is the system under consideration which goes choosing the correlation effects between blocks during the renormalization process.

We have tested the CBRG method on the 1D and 2D Tight-Binding model with different boundary conditions and obtained very good results for the spectrum of low lying states and its real space representation of their wave functions. Moreover, we have correctly reproduced the scaling laws $n^{2} / N^{2}$ for the energy of the excited states in 1D for Free-Free, Free-Fixed and Fixed-Fixed BC's and the scaling law $\left(n_{1}^{2}+n_{2}^{2}\right) / N^{2}$ for Free BC's in 2D. In addition, the correct dynamical exponent $z$ is also reproduced. Therefore, we believe that we have definitely solved in a transparent and systematic way the boundary problem for Tight-Binding models posed by Wilson [6] in his search for a better understanding of how a Real-Space RG method works.

In order to have a more extensive comprenhension of the correlations introduced in our CBRG method, we think it would be enlightning to make a thorough comparison between the process of truncation of states in our method and the one employed in the DMRG method by means of the onionscheme. This issue is left for future work. Another important line of study is the extension of the present CBRG method to interacting systems appearing in many-body problems. In this regard, we are currently studying a many-body system of fermions in one dimension as another prospective use of our method.

\section{Acknowledgements}

One of us (G.S.) would like to thank T. Nishino for useful discussions.

Work partially supported in part by CICYT under contracts AEN93-0776 (M.A.M.-D.) and the Swiss National Science Foundation and by the Spanish Fund DGICYT, Ref. PR95-284 (G.S.). 


\section{References}

[1] K.R. Wilson, Rev. Mod. Phys. 47, 773 (1975).

[2] S.D. Drell, M. Weinstein, S. Yankielowicz, Phys. Rev. D 16, 1769 (1977).

[3] R. Jullien, P. Pfeuty, J.N. Fields, S. Doniach, Phys. Rev. B 18, 3568 (1978).

[4] S.R. White, Phys. Rev. Lett. 69, 2863 (1992); Phys. Rev. B 48, 10345 (1993).

[5] S.R. White, R.M. Noack, Phys. Rev. Lett. 68, 3487 (1992).

[6] K.R. Wilson in unpublished talk.

[7] S.R. White and D.A. Huse Phys. Rev. B 48, 3844 (1993).

[8] R.M.Noack, S.R. White and D.J. Scalapino Phys. Rev. Lett. 73, 882 (1994).

[9] T. Nishino and K. Okunishi, "Corner Transfer Matrix Renormalization Group Method" Preprint 1995 Tohoku University. cond-mat/9507087.

[10] S. Ostlund and S. Rommer, "Thermodynamic limit of the density matrix renormalization for the spin-1 Hesisenberg chain". Preprint March 1995, Goteborg ITP 95-6.

[11] T. Nishino and K. Okunishi, "Product Wave Function Renormalization Group". Preprint 1995 Tohoku University. cond-mat/9510004.

[12] M.A. Martín-Delgado and G. Sierra, 1995, UCM-CSIC preprint, "Real Space Renormalization Group Methods and Quantum Groups" to appear in Phys. Rev. Lett.

[13] M.A. Martín-Delgado and G. Sierra, 1995, UCM-CSIC preprint, to appear in "From Field Theory to Quantum Groups". World Scientific Publishers.

[14] M.A. Martín-Delgado and G. Sierra, 1995, UCM-CSIC preprint, "Analytic Formulations of the Density Matrix Renormalization Group", to be appear in Int. J. Mod. Phys. A.

[15] M.A. Martín-Delgado and G. Sierra, 1995, UCM-CSIC preprint, "The Role of Boundary Conditions in the Real-Space Renormalization Group". Phys. Lett. B364 41, (1995).

[16] A. Fernández-Pacheco, Phys. Rev. D 19, 3173 (1979).

[17] J.M. Rabin, Phys. Rev. B 21, 2027 (1980).

[18] Pfeuty, P.Jullien, R. and Penson, K.A., in "Real-Space Renormalization", editors Burkhardt, T.W. and van Leeuwen, J.M.J., series topics in Current Physics 30, Springer-Verlag 1982.

[19] J. González, M.A. Martín-Delgado, G. Sierra, A.H. Vozmediano, Quantum Electron Liquids and High- $T_{c}$ Superconductivity, Lecture Notes in Physics, Monographs vol. 38, Springer-Verlag 1995. 


\section{Table captions}

Table 1 : Exact and CBRG Values of Low Lying States for the 1D Tight-Binding Model for a chain of $N=12 \times 2^{6}=768$ sites with Free-Free BC's.

Table 2 : Exact and new CBRG values of the first excited state for the 1D Tight-Binding Model with Free-Free BC's.

Table 3 : Exact, Standard RG and CBRG Values of Low Lying States for the 1D Tight-Binding Model for a chain of $N=12 \times 2^{5}=384$ sites with Free-Fixed BC's.

Table 4 : Exact, Standard RG and CBRG Values of Low Lying States for the 1D Tight-Binding Model for a chain of $N=12 \times 2^{5}=384$ sites with Fixed-Fixed BC's.

Table 5 : Exact and CBRG Values of Low Lying States for the 2D Tight-Binding Model for a lattice of $N=4 \times 4 \times 4^{6}=65536$ sites with Free BC's. 


\section{Figure captions}

Figure 1: Pictorical decomposition of a given Hamiltonian $H$ into uncorrelated $A$-operators, cor-

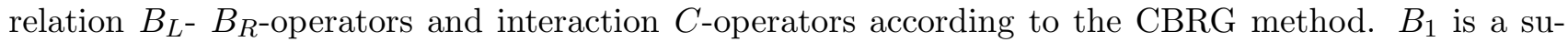
perblock made up of two $L_{1}$ and $R_{1}$ blocks.

Figure 2 : The $n^{2} / N^{2}$-law for the first 5 excited states of the 1D Tight-Binding Model for a chain of $N=12 \times 2^{m}$ sites with Free-Free BC's. This is a $\ln E_{n}-\ln m$ plot.

Figure 3 : The wave function reconstruction for the first 5 excited states of the 1D Tight-Binding Model for a chain of $N=12 \times 2^{6}=768$ sites with Free-Free BC's. We have scaled up the exact results by a factor of 1.23 for clarity.

Figure 4 : Pictorical representation of uncorrelated $A$-operators, correlation $B_{L^{-}} B_{R^{-}} B_{U} B_{D^{-}}$ operators and interaction $C_{L R^{-}} C_{U D^{-o p e r a t o r s}}$ according to the CBRG method in $\mathrm{D}=2$ dimensions.

Figure 5 : a) Two-dimensional superblock decomposition into 4 blocks of $2 \times 2$ sites showing the choice of basis. b) Vertical and horizontal interactions between 4 neighbouring superblocks in $\mathrm{D}=2$ dimensions.

Figure 6 : Vertical and horizontal renormalizations of neighbouring superblocks in $\mathrm{D}=2$ dimensions.

Figure 7 : Plot of the wave function reconstruction for the singlet $(1,1)$-excited state in the 2D Tight-Binding Model for a lattice of $N=4 \times 4 \times 4^{6}=65536$ sites with Free BC's. 


\begin{tabular}{|c|c|c|}
\hline \hline Energies & Exact & CBRG \\
\hline \hline$E_{0}$ & 0 & $1.1340 \times 10^{-14}$ \\
\hline$E_{1}$ & $1.6733 \times 10^{-5}$ & $1.9752 \times 10^{-5}$ \\
\hline$E_{2}$ & $6.6932 \times 10^{-5}$ & $7.6552 \times 10^{-5}$ \\
\hline$E_{3}$ & $1.5060 \times 10^{-4}$ & $1.8041 \times 10^{-5}$ \\
\hline$E_{4}$ & $2.6772 \times 10^{-4}$ & $2.9681 \times 10^{-4}$ \\
\hline$E_{5}$ & $4.1831 \times 10^{-4}$ & $5.1078 \times 10^{-4}$ \\
\hline \hline
\end{tabular}

Table 1: Exact and CBRG Values of Low Lying States for the 1D Tight-Binding Model for a chain of $N=12 \times 2^{6}=768$ sites with Free-Free BC's.

\begin{tabular}{|c|c|c|c|}
\hline \hline $\mathrm{m}$ & $\mathrm{N}=122^{m}$ & $E_{1}^{(\text {exact })}(N)$ & $E_{1}^{(C B R G)}(N)$ \\
\hline \hline 0 & 12 & $6.8148 \times 10^{-2}$ & $6.8148 \times 10^{-2}$ \\
\hline 1 & 24 & $1.7110 \times 10^{-2}$ & $1.7375 \times 10^{-2}$ \\
\hline 2 & 48 & $4.2826 \times 10^{-3}$ & $4.4694 \times 10^{-3}$ \\
\hline 3 & 96 & $1.0708 \times 10^{-3}$ & $1.1515 \times 10^{-3}$ \\
\hline 4 & 192 & $2.6772 \times 10^{-4}$ & $2.9681 \times 10^{-4}$ \\
\hline 5 & 384 & $6.6932 \times 10^{-5}$ & $7.6552 \times 10^{-5}$ \\
\hline 6 & 768 & $1.6733 \times 10^{-5}$ & $1.9752 \times 10^{-5}$ \\
\hline & $\gg 1$ & $\pi^{2} / N^{2}$ & $9.8080 / N^{2}$ \\
\hline \hline
\end{tabular}

Table 2: Exact and new CBRG values of the first excited state for the 1D Tight-Binding Model with Free-Free BC's.

\begin{tabular}{|c|c|c|c|}
\hline \hline Energies & Exact & Standard BRG & CBRG \\
\hline \hline$E_{0}$ & $1.7754 \times 10^{-5}$ & $1.5771 \times 10^{-2}$ & $1.8409 \times 10^{-5}$ \\
\hline$E_{1}$ & $1.5043 \times 10^{-4}$ & $4.2679 \times 10^{-2}$ & $1.6655 \times 10^{-4}$ \\
\hline$E_{2}$ & $4.1761 \times 10^{-4}$ & $4.2794 \times 10^{-2}$ & $4.6408 \times 10^{-4}$ \\
\hline$E_{3}$ & $8.1831 \times 10^{-4}$ & $4.3053 \times 10^{-2}$ & $9.1450 \times 10^{-4}$ \\
\hline$E_{4}$ & $1.3520 \times 10^{-3}$ & $4.3173 \times 10^{-2}$ & $1.5179 \times 10^{-3}$ \\
\hline$E_{5}$ & $2.0196 \times 10^{-3}$ & $4.4288 \times 10^{-2}$ & $2.2852 \times 10^{-3}$ \\
\hline \hline
\end{tabular}

Table 3: Exact, Standard RG and CBRG Values of Low Lying States for the 1D Tight-Binding Model for a chain of $N=12 \times 2^{5}=384$ sites with Free-Fixed BC's.

\begin{tabular}{|c|c|c|c|}
\hline \hline Energies & Exact & Standard BRG & CBRG \\
\hline \hline$E_{0}$ & $6.6585 \times 10^{-5}$ & $5.8116 \times 10^{-2}$ & $7.0843 \times 10^{-5}$ \\
\hline$E_{1}$ & $2.6633 \times 10^{-4}$ & $5.8155 \times 10^{-2}$ & $2.9403 \times 10^{-4}$ \\
\hline$E_{2}$ & $5.9924 \times 10^{-4}$ & $5.8268 \times 10^{-2}$ & $6.3690 \times 10^{-4}$ \\
\hline$E_{3}$ & $1.0653 \times 10^{-3}$ & $5.8470 \times 10^{-2}$ & $1.2289 \times 10^{-3}$ \\
\hline$E_{4}$ & $1.6644 \times 10^{-3}$ & $5.8717 \times 10^{-2}$ & $1.7707 \times 10^{-3}$ \\
\hline$E_{5}$ & $2.3966 \times 10^{-3}$ & $5.9106 \times 10^{-2}$ & $2.7311 \times 10^{-3}$ \\
\hline \hline
\end{tabular}

Table 4: Exact, Standard RG and CBRG Values of Low Lying States for the 1D Tight-Binding Model for a chain of $N=12 \times 2^{5}=384$ sites with Fixed-Fixed BC's. 


\begin{tabular}{|c|c|c|}
\hline \hline Energies & Exact & CBRG \\
\hline \hline$E_{0}$ & 0 & $9.6114 \times 10^{-35}$ \\
\hline$E_{1}$ & $1.5056 \times 10^{-4}$ & $1.9390 \times 10^{-4}$ \\
\hline$E_{2}$ & $1.5056 \times 10^{-4}$ & $1.9390 \times 10^{-4}$ \\
\hline$E_{3}$ & $3.0012 \times 10^{-4}$ & $3.8781 \times 10^{-4}$ \\
\hline \hline
\end{tabular}

Table 5: Exact and CBRG Values of Low Lying States for the 2D Tight-Binding Model for a lattice of $N=4 \times 4 \times 4^{6}=65536$ sites with Free BC's. 